\title{
$\beta$-Oxo Anilides in Heterocyclic Synthesis: Novel Synthesis of Pyridazinones, Pyrazolopyridazines and Cinnolines
}

\author{
A. M. Hussein ${ }^{1}$, M. S. A. El-Gaby ${ }^{1}$, F. A. Abu-Shenab ${ }^{1}$, M. A. M. Abdel-Raheim ${ }^{1}$ \\ and M. A. El-Apasery ${ }^{2 *}$ \\ ${ }^{1}$ Department of Chemistry, Faculty of Science, Al Azhar University, Assiut 71524, \\ Egypt. \\ ${ }^{2}$ Dyeing, Printing and Textile Auxiliaries Department, Textile Research Division, \\ National Research Centre, 33 El Buhouth St., Dokki, Cairo, Egypt.
}

\begin{abstract}
COMPOUND 1 coupled smoothly with aromatic diazonium salts to yield the corresponding arylhydrazones $2 \mathrm{a}-\mathrm{d}$. Compounds $2 \mathrm{a}-\mathrm{d}$ condensed with DMF-DMA in refluxing xylene to yield the pyridazinones 3a-d. Compounds 3a-d were also established based on its further reaction with some active methylene reagents and some nucleophilic reagents. So, reactions of $3 \mathrm{a}, \mathrm{b}$ with malononitrile in refluxing ethanolic piperidine afforded arylidinemalononitrile $4 \mathrm{a}, \mathrm{b}$. The pyridazinone derivatives $3 \mathrm{a}$, b reacted with hydrazine hydrate to afford the hydrazine derivatives $5 \mathrm{a}, \mathrm{b}$. When $3 \mathrm{a}, \mathrm{b}$ were fused with hydrazine hydrate without solvent, the pyrazolo[4,3-c]-pyridazines $6 \mathrm{a}, \mathrm{b}$ were obtained. Compounds $6 \mathrm{a}, \mathrm{b}$ were also obtained when compounds $5 \mathrm{a}, \mathrm{b}$ melt over melting point for short time. Condensation of $2 \mathrm{a}, \mathrm{b}$ with ethyl cyanoacetate yield $7 \mathrm{a}, \mathrm{b}$. Similarly, reactions of $2 \mathrm{a}, \mathrm{b}$ with 1 mole of malononitrile afforded the pyridazine derivatives 8a,b. While, two moles of malononitrile reacted with $2 \mathrm{a}, \mathrm{b}$ in the same experimental conditions to yield the cinnoline derivative $9 \mathrm{a}, \mathrm{b}$. Reactions of pyridazine $8 \mathrm{a}, \mathrm{b}$ with 1 mole of malononitrile afforded 9 . Compound $2 \mathrm{~b}$ was reacted with a mixture of arylidinemalononitrile and acrylonitrile to yield product formulated as triazole moieties $12 \mathrm{a}$, b. Similarly compound $2 \mathrm{~b}$ was reacted with a mixture of maleic anhydride and acrylonitrile in the same above experimental conditions to give 13. Also, reactions of $2 \mathrm{~b}$ with hydroxylamine hydrochloride yield 14 .
\end{abstract}

Keywords: Pyridazinones, Dyes, Pyrazolopyridazines and Cinnolines.

\section{Introduction}

Polyfunctionally substituted heteroaromatics are interesting as potential agrochemicals [1-3], pharmaceuticals $[4,5]$ and intermediates for the preparation of dyes [6, 7]. Specially condensed pyridazinones are biologically interesting molecules and their chemistry is now receiving considerable interest [8-15]. In conjunction with our interest in the synthesis of azines and condensed azines [16-18]. New route to pyridazinones has been discovered in our laboratory. This route enabled the reactivity of $\beta$-oxo anilide 1 toward aromatic and heteroaromatic diazonium salt as a route to new azines and condensed azines of potential activity as dyes, dye intermediates and agrochemicals.

\section{$\underline{\text { Results and Discussions }}$}

$\beta$-Oxo anilide 1 coupled smoothly with aromatic diazonium salts in ethanolic sodium acetate to yield the corresponding arylhydrazones 2a-d in good yield $[19,20]$, these compounds could be also used as disperse dyes [21]. Compounds 2a-d condensed with DMF-DMA in refluxing xylene to yield the pyridazinones $3 \mathrm{a}-\mathrm{d}$ in excellent yield (Scheme 1).

These structures were established on the basis of spectral analysis as well as elemental analysis. ${ }^{1} \mathrm{H}$ NMR spectrum of compound 3a as an example showed two douplet signals in the range of $\delta 7.63-7.65 \mathrm{ppm}$ and $\delta 7.82-7.84$ ppm corresponding to the two pyridazine $\mathrm{CH}$ protons (H-5 and $\mathrm{H}-6)$ respectively. Compounds 3a-d were also established based on its further

*Corresponding author: Morsy A. El-Apasery; E-mail: elapaserym@yahoo.com

DOI: 10.21608/ejchem.2018.3901.1343

C2017 National Information and Documentation Center (NIDOC) 
reaction with some active methylene reagents and some nucleophilic reagents. So, reactions of $3 \mathrm{a}, \mathrm{b}$ with malononitrile in refluxing ethanolic piperidine afforded arylidinemalononitrile $4 \mathrm{a}, \mathrm{b}$ (Scheme 1). These compounds were established based on its spectroscopic analysis. The IR spectrum of compound $4 \mathrm{a}$ showed characteristic bands at $2200 \mathrm{~cm}^{-1}$ of two $\mathrm{CN}$ groups and disappearance of carbonyl group at $\delta 1698$ of compound $3 \mathrm{a}$.

The pyridazinone derivatives $3 \mathrm{a}$, b reacted with hydrazine hydrate in refluxing ethanol to afford the hydrazine derivatives 5a, b (Scheme 1). Compounds $5 \mathrm{a}, \mathrm{b}$ were established based on its elemental analysis and spectroscopic data. IR spectrum for $5 \mathrm{a}$ as an example showed the presence of $\mathrm{NH}_{2}$ group at $v 3340,3300 \mathrm{~cm}^{-1}$ beside $\mathrm{NH}$ group at $3184 \mathrm{~cm}^{-1}$ and disappearance of carbonyl group (CO) of compound (3a). Also, the ${ }^{1} \mathrm{H}$ NMR spectrum of compound (5a) showed the presence of broad signal at $\delta 4.32$ ppm for protons of $\mathrm{NH}_{2}$ and deuterable signal at $\delta 12.62 \mathrm{ppm}$ for one proton $(\mathrm{NH})$.<smiles>CC(=O)CC(=O)Nc1nc2ccccc2s1</smiles>

(1)<smiles>CC(=O)/C(=N\N[Al])C(=O)Nc1nc2ccccc2s1</smiles>

(2a-d)<smiles></smiles>

\author{
2,3-a; $\mathrm{Ar}=\mathrm{C}_{6} \mathrm{H}_{4} \mathrm{Cl}(\mathrm{p})$ \\ b; $\mathrm{Ar}=\mathrm{C}_{6} \mathrm{H}_{4} \mathrm{OMe}(\mathrm{p})$ \\ c; $\mathrm{Ar}=\mathrm{C}_{6} \mathrm{H}_{4} \mathrm{Br}(\mathrm{p})$ \\ $\mathrm{d} ; \mathrm{Ar}=$ benzenothiazole
}

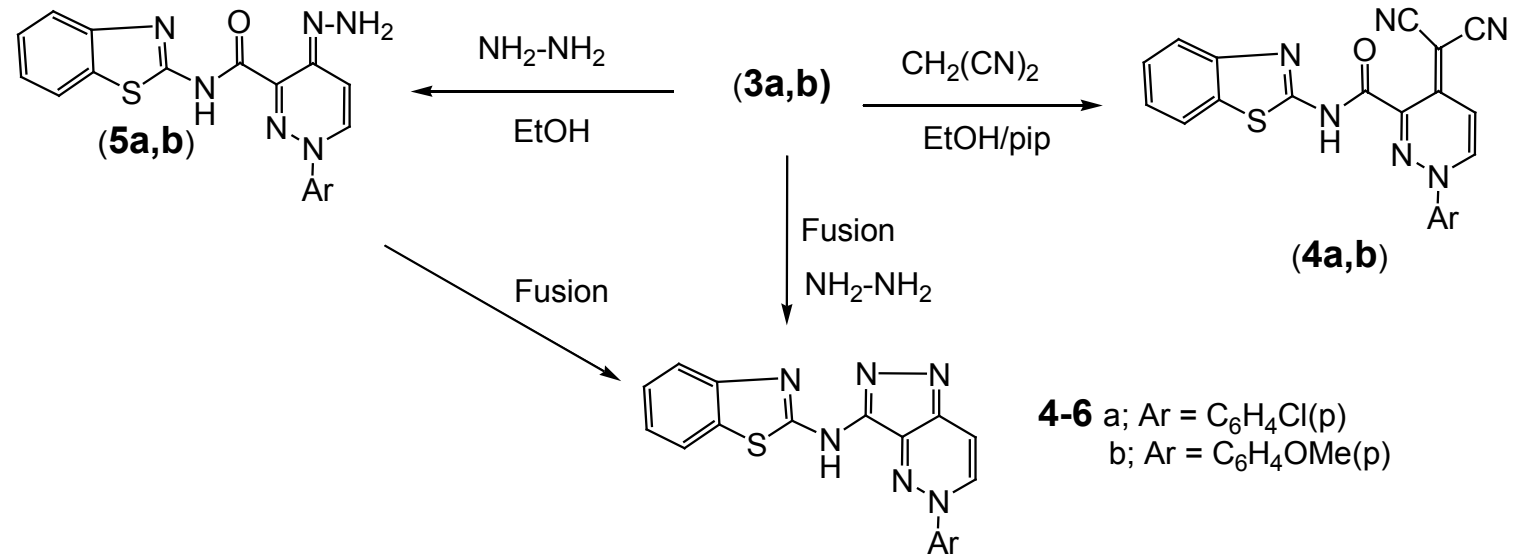

$(6 a, b)$

Scheme 1. 
When 3a, b were fused with hydrazine hydrate without solvent, the pyrazolo[4,3-c]pyridazines $6 \mathrm{a}, \mathrm{b}$ were obtained. Compounds $6 \mathrm{a}, \mathrm{b}$ were also obtained when compounds $5 \mathrm{a}$, b melt over melting point for short time (Scheme 1). The IR spectrum of compound $6 \mathrm{a}$ as an example showed the disappearance of $\mathrm{NH}_{2}$ and $\mathrm{CO}$ bands. Also, the ${ }^{1} \mathrm{H}$ NMR spectrum of compound 6a revealed only the two protons of pyridazine, aromatic protons and $\mathrm{NH}$ protons at $\delta=7.03,7.96$ and $10.73 \mathrm{ppm}$ respectively beside the aromatic protons at $\delta=$ 7.11-7.78.
Condensation of $2 \mathrm{a}, \mathrm{b}$ with ethyl cyanoacetate proceeded very readily either by fusion in the presence of ammonium acetate or by refluxing in the presence of the latter reagent in benzene utilizing a water separator for 5 hours to yield $7 \mathrm{a}, \mathrm{b}$ (Scheme 2). Compounds 7a,b were established based on spectral data (IR, ${ }^{1} \mathrm{H}$ NMR) and elemental analysis. The IR spectrum of compound $7 \mathrm{a}$ as an example revealed a CN band at $v 2199 \mathrm{~cm}^{-1}$, two carbonyl at $v 1670$ and $1630 \mathrm{~cm}^{-1}$. Also, ${ }^{1} \mathrm{H}$ NMR spectrum of that compound revealed singlet signal at $\delta 2.19 \mathrm{ppm}$ assigned to $\mathrm{CH}_{3}$, multiplet signals at $\delta$ 7.28-7.95 ppm corresponding to $\mathrm{Ar}-\mathrm{H}$, and singlet signal at $\delta 12.29 \mathrm{ppm}$ assigned to $\mathrm{NH}$.

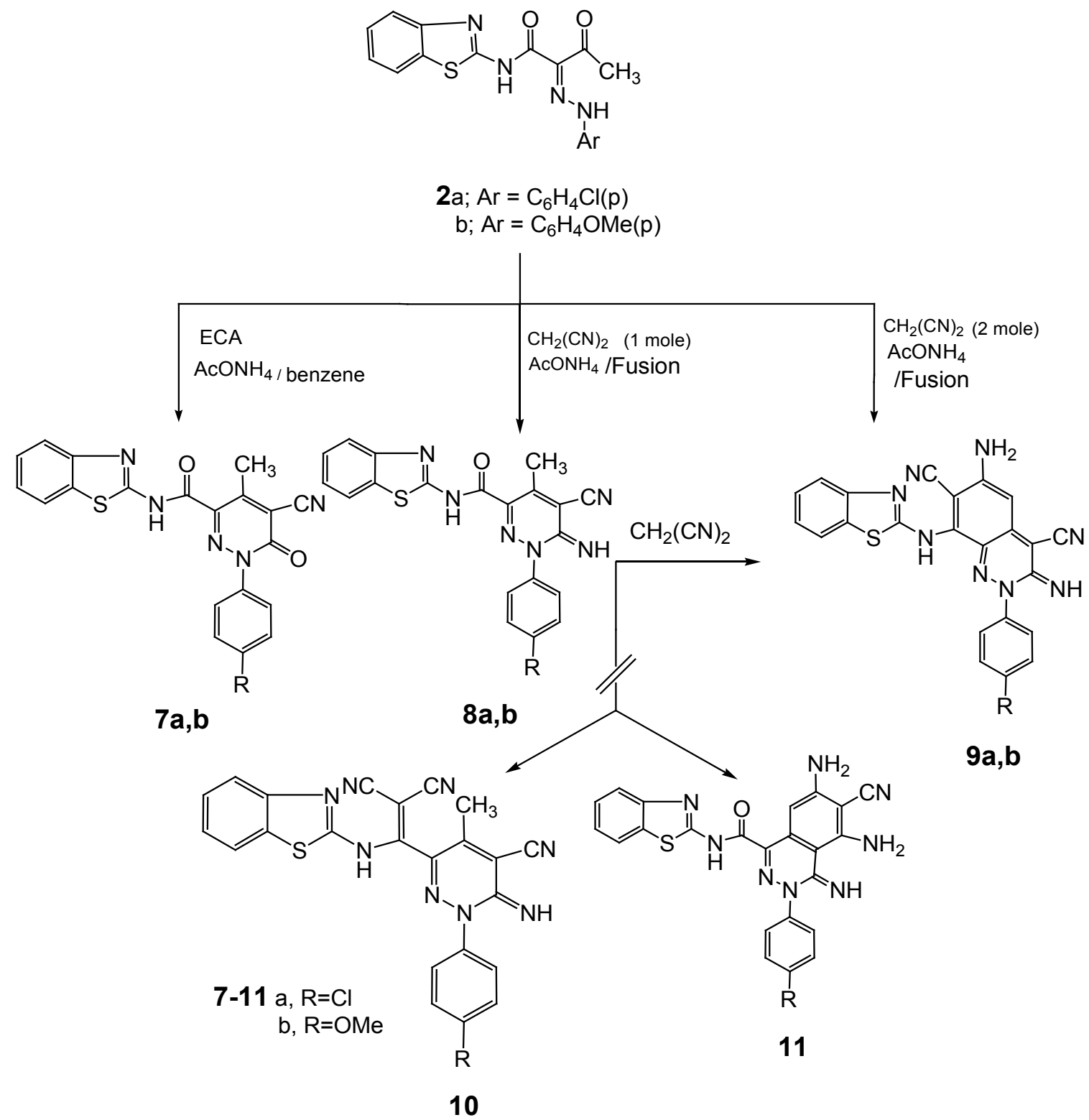

Scheme 2. 
Similar to this reaction, reactions of $2 a, b$ with one mole of malononitrile afforded the pyridazine derivatives $8 \mathrm{a}$, $\mathrm{b}$ which were established based on its spectral data and elemental analysis, while, when two moles of malononitrile was reacted with 2a,b in the same experimental conditions, cinnoline derivatives $9 \mathrm{a}, \mathrm{b}$ were formed. These compounds were established based on its spectroscopic data and elemental analysis. The infrared spectrum of compound 9a as an example was characterized by the presence of amino function groups at 3400, 3341 and $3194 \mathrm{~cm}^{-1}$, cyano function group at $2202 \mathrm{~cm}^{-1}$ and disappearance of carbonyl function group. Reactions of pyridazine $8 \mathrm{a}, \mathrm{b}$ with (one mole) of malononitrile in presence of ammonium acetate in oil bath without solvent, three possible structures 9, 10 and 11 can be formed. Structures 10,11 were eliminated on the basis of analytical and spectral data. Establishing the exact structure of the reaction product as structure 9 based on the comparison of spectroscopic data, also, the IR spectrum of product don't show band of $\mathrm{C}=\mathrm{O}$ group at range $1725-1660 \mathrm{~cm}^{-1}$ characteristic to phthalazine derivative 11 (Scheme 2).

The behaviour of arylhydrazone 2 towards $\alpha, \beta$-unsaturated nitrile and nucleophilic reagents was also investigated. Thus, compound $2 \mathrm{~b}$ was reacted with a mixture of arylidinemalononitrile and acrylonitrile in oil bath without solvent and in presence of ammonium acetate to yield product which was formulated as triazole moities $12 \mathrm{a}, \mathrm{b}$ (Scheme 3). Compounds 12a, b were compatible establishing based on its elemental analysis and spectral data. The IR spectrum of $12 \mathrm{a}$ as an example showed the appearance of $\mathrm{CN}$ group at $\checkmark 2201 \mathrm{~cm}^{-1}$. Also, ${ }^{1} \mathrm{H}$ NMR spectrum revealed a singlet signal for $\mathrm{NH}$ group at $\delta 8.77 \mathrm{ppm}$, olefinic $(\mathrm{CH})$ at $\delta 5.93 \mathrm{ppm}$.

Similarly compound $2 \mathrm{~b}$ was reacted with a mixture of maleic anhydride and acrylonitrile in the same above experimental conditions to give compound 13. Also, reactions of $2 b$ with hydroxylamine hydrochloride in refluxing ethanol containing few grams of sodium acetate yield the hydrazone derivative 14 [21]. The structure of isoxazole derivatives 14 was established based on spectroscopic data (IR and ${ }^{1} \mathrm{H}$ NMR). Thus, IR spectrum showed the disappearance of two carbonyl group $(2 \mathrm{C}=\mathrm{O})$. Also, the ${ }^{1} \mathrm{H}$ NMR spectrum exhibited a singlet signal at $\delta 2.48 \mathrm{ppm}$ assigned to $\mathrm{CH}_{3}$, singlet signal at $\delta 3.80 \mathrm{ppm}$ assigned to $\mathrm{OCH}_{3}$, multiplet signals at $\delta$ 6.997.99 coresponding to $\mathrm{Ar}-\mathrm{H}$, and singlet signal at $\delta$ $12.71 \mathrm{ppm}$ assigned to NH. (Scheme 3 ).



Egypt. J. Chem. 61, No. 6 (2018)

Scheme 3 . 


\section{Materials and Methods}

All melting points are uncorrected. IR spectra $(\mathrm{KBr})$ were recorded on a FTIR 5300 spectrometer $\left(v, \mathrm{~cm}^{-1}\right)$. The ${ }^{1} \mathrm{H}$ NMR spectra were recorded in DMSO-d $\mathrm{d}_{6}$ and $\mathrm{CDCl}_{3}$ at 200, $400 \mathrm{MHz}$ on a Varian Gemini NMR spectrometer $(\delta$, ppm) using TMS as an internal standard. Mass spectra were obtained on GC Ms-QP 1000 EX mass spectrometer at 70 ev. Elemental analysis was carried out by the Microanalytical Research Center, Faculty of Science, Cairo University and Microanalytical Research Center, Assiut University.

Preparation of Compounds (2a-d): General Procedure

A solution of (1) (0.01 mole) in ethanol (100 $\mathrm{ml})$ containing sodium acetate $(2.0 \mathrm{~g})$ was cooled to $0^{\circ} \mathrm{c}$, stirred and treated gradually with a cooled solution of aryldiazonium chloride (prepared from 0.01 mole of amine and the appropriate quantities of $\mathrm{HCl}$ and $\mathrm{NaNO}_{2}$ ). The solid product formed on standing was collected and recrystallized from the appropriate solvent to give (2a-d).

$N$-1,3-Benzothiazol-2-yl-2-[(4-chloro-phenyl)hydrazono]-3-oxo-butanamide (2a)

It was obtained as yellow crystals from dioxane/ethanol; yield $90 \%$; m.p. $260^{\circ} \mathrm{C}$; IR $(\mathrm{KBr}) \vee \mathrm{cm}^{-1} 3420,3240(2 \mathrm{NH}), 3075$ (CHarom), 2965 (CH-aliph), 1650, $1630(2 \mathrm{C}=\mathrm{O}) ;{ }^{1} \mathrm{H}$ NMR $\left(\mathrm{CDCl}_{3}\right) \delta=2.57\left(\mathrm{~s}, 3 \mathrm{H}, \mathrm{CH}_{3}\right), 7.24-7.85$ (m, 8H, Ar-H), 12.70 (s, 1H, NH), 14.32 (s, 1H, NH). Found; C, 54.78; H, 3.53; N, 15.04; cald. For $\mathrm{C}_{17} \mathrm{H}_{13} \mathrm{ClN}_{4} \mathrm{O}_{2} \mathrm{~S}$ (372.84): C, 54.77; H, 3.51; N, 15.03.

N-1,3-Benzothiazol-2-yl-2-[(4-methoxy-phenyl)hydrazono]-3-oxo-butanamide (2b)

It was obtained as yellow crystals from dioxane/EtOH; yield 87\%; m.p. $216^{\circ} \mathrm{C}$; IR ( $\mathrm{KBr}$ ) $v \mathrm{~cm}^{-1}$ 3178, 3142 (2NH), 3063 (CH-arom), 2916, 2863 (CH-aliph), 1718, $1660(2 \mathrm{C}=\mathrm{O}) ;{ }^{1} \mathrm{H}$ NMR $\left(\mathrm{DMSO}_{6}\right) \delta=2.60\left(\mathrm{~s}, 3 \mathrm{H}, \mathrm{CH}_{3}\right), 3.86(\mathrm{~s}, 3 \mathrm{H}$, $\left.\mathrm{OCH}_{3}\right), 6.97-7.89(\mathrm{~m}, 8 \mathrm{H}, \mathrm{Ar}-\mathrm{H}), 12.82(\mathrm{~s}, 1 \mathrm{H}$, $\mathrm{NH}), 14.46$ (s, 1H, NH). Found; C, 58.69; H, 4.37; N, 15.23; cald. For $\mathrm{C}_{18} \mathrm{H}_{16} \mathrm{~N}_{4} \mathrm{O}_{3} \mathrm{~S}$ (368.42): C, 58.68; H, 4.38; N, 15.21.

\section{N-1,3-Benzothiazol-2-yl-2-[(4-bromo-phenyl)-} hydrazono]-3-oxo-butanamide (2c)

It was obtained as yellow crystals from ethanol; yield $80 \%$; m.p. $200^{\circ} \mathrm{C}$; IR $(\mathrm{KBr}) \vee \mathrm{cm}^{-1}$ 3301, 3177 (2NH), 3063 (CH-arom), 2920 (CHaliph), 1715, 1658 (2C=O). Found; C, 48.95; H,
3.15 ; N, 13.45; cald. For $\mathrm{C}_{17} \mathrm{H}_{13} \mathrm{BrN}_{4} \mathrm{O}_{2} \mathrm{~S}$ (417.29): C, 48.93; H, 3.14; N, 13.43 .

N-1,3-Benzothiazol-2-yl-2-(1,3-benzothiazol-2yl-hydrazono)-3-oxo-butanamide (2d)

It was obtained as brown crystals from dioxane/EtOH; yield $86 \%$; m.p. $214^{\circ} \mathrm{C}$; IR $(\mathrm{KBr})$ $v \mathrm{~cm}^{-1} 3415,3240(2 \mathrm{NH}), 3070 \quad(\mathrm{CH}-$ arom $)$, 2965 (CH-aliph), 1715, 1659 (2C=O). Found; C, 54.68; H, 3.33; N, 17.73; cald. For $\mathrm{C}_{18} \mathrm{H}_{13} \mathrm{~N}_{5} \mathrm{O}_{2} \mathrm{~S}_{2}$ (395.46): C, 54.67; H, 3.31; N, 17.71.

\section{Preparation of Compounds (3a-d): General Procedure}

Equimolar amounts of DMF-DMA (0.01 mole) and compounds (2a-d) (0.01 mole) in dry xylene $(30 \mathrm{ml})$ were refluxed for $6 \mathrm{hr}$. The reaction mixture was left to stand at room temperature. The product formed was collected by filtration and recrystallized from the proper solvent to give (3a-d).

N-1,3-Benzothiazol-2-yl-1-(4-chlorophenyl)-4oxo-1,4-dihydropyridazine-3-carboxamide (3a)

It was obtained as brown crystals from DMF/ ethanol; yield $70 \%$; m.p. $>300^{\circ} \mathrm{C}$; IR $(\mathrm{KBr}) v$ $\mathrm{cm}^{-1} 3470(\mathrm{NH}), 1698,1651(2 \mathrm{C}=\mathrm{O}) ;{ }^{1} \mathrm{H}$ NMR $\left(\right.$ DMSO-d $\left._{6}\right) \delta=7.05-7.45$ (m, 9H, Ar-H and NH), 7.63-7.65 (d, 1H, pyridazine-H) $\mathrm{J}=8.2(\mathrm{~Hz}), 7.82-$ 7.84 (d, 1H, pyridazine-H) J = $8.0(\mathrm{~Hz})$. Found; C, 56.48; H, 2.91; N, 14.62; cald. For $\mathrm{C}_{18} \mathrm{H}_{11} \mathrm{ClN}_{4} \mathrm{O}_{2} \mathrm{~S}$ (382.83): C, 56.47; H, 2.90; N, 14.63.

N-1,3-Benzothiazol-2-yl-1-(4-methoxyphenyl)-4oxo-1,4-dihydropyridazine-3-carboxamide (3b)

It was obtained as brown crystals from $\mathrm{DMF} / \mathrm{EtOH}$; yield $80 \%$; m.p. $270-2^{\circ} \mathrm{C}$; IR $(\mathrm{KBr}) \vee \mathrm{cm}^{-1} 3427(\mathrm{NH}), 3037$ (CH-arom), 2922 (CH-aliph), 1644, $1630(2 \mathrm{C}=\mathrm{O}) ;{ }^{1} \mathrm{H}$ NMR $\left(\mathrm{DMSO}_{\mathrm{d}}\right) \delta=3.80\left(\mathrm{~s}, 3 \mathrm{H}, \mathrm{OCH}_{3}\right), 7.03-7.79$ (m, 8H, Ar-H), 7.83-7.85 (d, 1H, pyridazine-H), 8.02-8.04 (d, 1H, pyridazine-H), $13.00(\mathrm{~s}, 1 \mathrm{H}$, $\mathrm{NH})$. Found; C, 60.32; H, 3.75; N, 14.82; cald. For $\mathrm{C}_{19} \mathrm{H}_{14} \mathrm{~N}_{4} \mathrm{O}_{3} \mathrm{~S}$ (378.41): C, 60.31; H, 3.73; $\mathrm{N}, 14.81$.

N-1,3-Benzothiazol-2-yl-1-(4-bromophenyl)-4oxo-1,4-dihydropyridazine-3-carboxamide (3c)

It was obtained as brown crystals from ethanol; yield $72 \%$; m.p. $220^{\circ} \mathrm{C}$; IR $(\mathrm{KBr})$ v $\mathrm{cm}^{-1} 3436$ (NH), 3050 (CH-arom), 1676, 1636 $(2 \mathrm{C}=\mathrm{O})$. Found; C, 50.62; H, 2.58; N, 13.13; cald. For $\mathrm{C}_{18} \mathrm{H}_{11} \mathrm{BrN}_{4} \mathrm{O}_{2} \mathrm{~S}$ (427.28): C, 50.60; H, $2.59 ; \mathrm{N}, 13.11$. 
N,1-bis (1,3-Benzothiazol-2-yl) -4- oxo-1,4dihydropyridazine -3-carboxamide (3d)

It was obtained as pale yellow crystals from ethanol; yield $66 \%$; m.p. $286^{\circ} \mathrm{C}$; IR $(\mathrm{KBr}) \vee \mathrm{cm}^{-1}$ $3422(\mathrm{NH}), 3050(\mathrm{CH}$-arom $), 1684,1670(2 \mathrm{C}=\mathrm{O})$; ${ }^{1} \mathrm{H}$ NMR (DMSO-d $) \delta=7.27-8.19$ (m, 8H, Ar$\mathrm{H}), 8.30-8.33(\mathrm{~d}, 1 \mathrm{H}$, pyridazine-H), $8.78(\mathrm{~s}, 1 \mathrm{H}$, NH), 8.93-8.95 (d, 1H, pyridazine-H). Found; C, 56.29; H, 2.75; N, 17.28; cald. For $\mathrm{C}_{19} \mathrm{H}_{11} \mathrm{~N}_{5} \mathrm{O}_{2} \mathrm{~S}_{2}$ (405.46): C, 56.28; H, 2.73; N, 17.27.

Preparation of Compounds (4a, b): General Procedure

A mixture of $(3 a, b) \quad(0.01$ mole $)$ and malononitrile $(0.01 \mathrm{~mole})$ in ethanol $(20 \mathrm{ml})$ was treated with a few drops of piperidine and refluxed for $4 \mathrm{hrs}$. The solid product formed was filtered off and recrystallized from the proper solvent to give $(4 a, b)$.

N-1,3-Benzothiazol-2-yl-1-(4-chlorophenyl)-4(dicyano-methylene)-1,4-dihydropyridazine-3carboxamide (4a)

It was obtained as buff crystals from DMF/ EtOH; yield $70 \%$; m.p. $310^{\circ} \mathrm{C}$; IR $(\mathrm{KBr}) \vee \mathrm{cm}^{-1}$ $3380(\mathrm{NH}), 3090$ (CH-arom), $2200(\mathrm{C} \equiv \mathrm{N}), 1655$ $(\mathrm{C}=\mathrm{O}) ;{ }^{1} \mathrm{H}$ NMR $\left(\mathrm{DMSO}_{6}\right) \delta=6.43(\mathrm{~d}, 1 \mathrm{H}$, pyridazine-H), 7.62-7.72 (m, 9H, Ar-H and NH), 8.74 (d, 1H, pyridazine-H). Found; C, 58.55; H, 2.58; N, 19.52; cald. For $\mathrm{C}_{21} \mathrm{H}_{11} \mathrm{ClN}_{6} \mathrm{OS}$ (430.88): C, 58.54; H, 2.57; N, 19.50 .

N-1,3-Benzothiazol-2-yl-4-(dicyanomethylene)1-(4-methoxy-phenyl)-1,4-dihydropyridazine-3carboxamide (4b)

It was obtained as brown crystals from ethanol; yield $64 \%$; m.p. $220^{\circ} \mathrm{C}$; IR $(\mathrm{KBr}) v$ $\mathrm{cm}^{-1} 3427(\mathrm{NH}), 2931$ (CH-aliph), $2202(\mathrm{C} \equiv \mathrm{N})$, $1651(\mathrm{C}=\mathrm{O}) ;{ }^{1} \mathrm{H}$ NMR $\left(\mathrm{DMSO}_{\mathrm{d}}\right) \delta=3.75(\mathrm{~s}$, $\left.3 \mathrm{H}, \mathrm{OCH}_{3}\right), 6.99-7.01(\mathrm{~d}, 1 \mathrm{H}$, pyridazine- $\mathrm{H})$, 7.25-7.78 (m, 9H, Ar-H and NH), 7.88-7.90 (d, $1 \mathrm{H}$, pyridazine-H). Found; C, 61.96; H, 3.32; N, 19.73; cald. For $\mathrm{C}_{22} \mathrm{H}_{14} \mathrm{~N}_{6} \mathrm{O}_{2} \mathrm{~S}$ (426.46): C, 61.96; H, 3.31; N, 19.71 .

Preparation of Compounds (5a, b): General Procedure

A mixture of $(3 a, b)(0.01$ mole $)$ and hydrazine hydrate $(0.02$ mole $)$ in ethanol $(20 \mathrm{ml})$ was refluxed for $2 \mathrm{hr}$. The reaction mixture was left to stand and the obtained product was recrystallized from the proper solvent to give $(5 a, b)$.
N-1,3-Benzothiazol-2-yl-1-(4-chlorophenyl)-4hydrazono-1,4-dihydropyridazine-3-carboxamide (5a)

It was obtained as buff crystals from ethanol; yield $66 \%$; m.p. $182^{\circ} \mathrm{C}$; IR $(\mathrm{KBr}) \vee \mathrm{cm}^{-1} 3340$, $3300\left(\mathrm{NH}_{2}\right), 3184(\mathrm{NH}), 3050(\mathrm{CH}$-arom), 1743 $(\mathrm{C}=\mathrm{O}) ;{ }^{1} \mathrm{H}$ NMR $\left(\right.$ DMSO-d $\left._{6}\right) \delta=4.32$ (hump, $2 \mathrm{H}$, $\left.\mathrm{NH}_{2}\right), 7.01$ (s, 1H, pyridazine-H), 7.30-7.42 (m, $8 \mathrm{H}, \mathrm{Ar}-\mathrm{H}), 7.90(\mathrm{~s}, 1 \mathrm{H}$, pyridazine-H), $12.62(\mathrm{~s}$, 1H, NH). Found; C, 54.49; H, 3.32; N, 21.19; cald. For $\mathrm{C}_{18} \mathrm{H}_{13} \mathrm{ClN}_{6} \mathrm{OS}$ (396.86): C, 54.48; H, $3.30 ; \mathrm{N}, 21.18$.

N-1,3-Benzothiazol-2-yl-4-hydrazono-1-(4methoxyphenyl)-1,4-dihydropyridazine-3carboxamide (5b)

It was obtained as brown crystals from $\mathrm{DMF} /$ $\mathrm{EtOH}$; yield $68 \%$; m.p. $>300^{\circ} \mathrm{C}$; IR $(\mathrm{KBr}) v$ $\mathrm{cm}^{-1}$ 3278, $3250\left(\mathrm{NH}_{2}\right), 3188(\mathrm{NH}), 3080(\mathrm{CH}-$ arom), 2919 (CH-aliph), $1660(\mathrm{C}=\mathrm{O})$. Found; C, 58.16; H, 4.13; N, 21.42; cald. For $\mathrm{C}_{19} \mathrm{H}_{16} \mathrm{~N}_{6} \mathrm{O}_{2} \mathrm{~S}$ (392.44): C, 58.15; H, 4.311; N, 21.41.

Preparation of Compounds $(6 a, b):$ General Procedure

Method (A)

A mixture of (3a,b) (0.01 mole) and excess of hydrazine hydrate $(10 \mathrm{ml})$ was refluxed for 24 hr. The solid product, so formed was collected by filtration and recrystallized from the proper solvent to give $(6 a, b)$.

Method (B)

Compounds $(5 \mathrm{a}, \mathrm{b})$ was fused for 10 minutes at over melting point. The reaction mixture was left to stand, then triturated with ethanol. The solid product so formed was collected by filtration and recrsytallized from the proper solvent to give $(6 a, b)$.

$\mathrm{N}$-1,3-Benzothiazol-2-yl-5-(4-chlorophenyl)-5Hpyrazolo-[4,3-c]pyridazin-3-amine (6a)

It was obtained as brown crystals from DMF/ EtOH; yield 67\%; m.p. $>300^{\circ} \mathrm{C}$; IR $(\mathrm{KBr}) v \mathrm{~cm}^{-1}$ $3425(\mathrm{NH}) ;{ }^{1} \mathrm{H}$ NMR $\left(\mathrm{DMSO}_{-}\right) \delta=7.03(\mathrm{~s}, 1 \mathrm{H}$, pyridazine-H), 7.11-7.78 (m, 8H, Ar-H), 7.96 (s, 1H, pyridazine-H), 10.73 (s, 1H, NH). Found; C, 57.08; H, 2.95; N, 22.19; cald. For $\mathrm{C}_{18} \mathrm{H}_{11} \mathrm{ClN}_{6} \mathrm{~S}$ (378.85): C, 57.07; H, 2.93; N, 22.18.

N-1,3-Benzothiazol-2-yl-5-(4-methoxyphenyl)5H-pyrazolo-[4,3-c]pyridazin-3-amine (6b)

It was obtained as brown crystals from DMF/ $\mathrm{EtOH}$; yield $70 \%$; m.p. $>300^{\circ} \mathrm{C}$; IR $(\mathrm{KBr}) \vee \mathrm{cm}^{-1}$ 
3410 (NH), 3035 (CH-arom), 2915 (CH-aliph). Found; C, 60.96; H, 3.78; N, 22.46; cald. For $\mathrm{C}_{19} \mathrm{H}_{14} \mathrm{~N}_{6} \mathrm{OS}$ (374.43): C, 60.95; H, 3.77; N, 22.45.

Preparation of Compounds (7a,b and $8 a, b)$ : General Procedure

A mixture of (2a,b) (0.01 mole), appropriate active methylene reagent (ethylcyanoacetate or malononitrile) ( 0.01 mole) and ammonium acetate $(2.0 \mathrm{gm})$ was fused for 30 minutes at $140^{\circ} \mathrm{C}$. The reaction mixture was left to stand, then triturated with ethanol. The solid product so formed was collected by filtration and recrystallized from the proper solvent to give $(7 \mathrm{a}, \mathrm{b}$ and $8 \mathrm{a}, \mathrm{b})$.

N-1,3-Benzothiazol-2-yl-1-(4-chlorophenyl)-5cyano-4-methyl-6-oxo-1,6-dihydropyridazine-3carboxamide (7a)

It was obtained as brown crystals from DMF/ EtOH; yield $76 \%$; m.p. $>300^{\circ} \mathrm{C}$; IR $(\mathrm{KBr}) \vee \mathrm{cm}^{-1}$ 3345 (NH), 2927 (CH-aliph), $2199(\mathrm{C} \equiv \mathrm{N}), 1670$, $1630(2 \mathrm{C}=\mathrm{O}) ;{ }^{1} \mathrm{H}$ NMR $\left(\mathrm{DMSO}_{6}\right) \delta=2.19(\mathrm{~s}$, $3 \mathrm{H}, \mathrm{CH}_{3}$ ), 7.28-7.95 (m, 8H, Ar-H), 12.29 (s, 1H, NH). Found; C, 56.95; H, 2.89; N, 16.62; cald. For $\mathrm{C}_{20} \mathrm{H}_{12} \mathrm{ClN}_{5} \mathrm{O}_{2} \mathrm{~S}$ (421.87): C, 56.94; H, 2.87; $\mathrm{N}, 16.60$.

N-1,3-Benzothiazol-2-yl-5-cyano-1-(4methoxyphenyl)-4-methyl-6-oxo-1,6dihydropyridazine-3-carboxamide (7b)

It was obtained as brown crystals from dioxane/ethanol; yield $80 \%$; m.p. $>300^{\circ} \mathrm{C}$; IR $(\mathrm{KBr}) \vee \mathrm{cm}^{-1} 3370(\mathrm{NH}), 2200(\mathrm{C} \equiv \mathrm{N}), 1660,1640$ $(2 \mathrm{C}=\mathrm{O}) ;{ }^{1} \mathrm{H}$ NMR $\left(\mathrm{DMSO}-\mathrm{d}_{6}\right) \delta=2.28(\mathrm{~s}, 3 \mathrm{H}$, $\left.\mathrm{CH}_{3}\right), 3.81$ (s, 3H, $\left.\mathrm{OCH}_{3}\right), 7.02-8.22(\mathrm{~m}, 9 \mathrm{H}, \mathrm{Ar}-\mathrm{H}$ and $\mathrm{NH}$ ). Found; C, 60.43; H, 3.64; N, 16.79; cald. For $\mathrm{C}_{21} \mathrm{H}_{15} \mathrm{~N}_{5} \mathrm{O}_{3} \mathrm{~S}$ (417.45): C, 60.42; H, $3.62 ; \mathrm{N}, 16.78$.

N-1,3-Benzothiazol-2-yl-1-(4-chlorophenyl)-5cyano-6-imino-4-methyl-1,6-dihydropyridazine3-carboxamide ( 8 a)

It was obtained as green crystals from dioxane/ ethanol; yield $71 \%$; m.p. $270-2^{\circ} \mathrm{C}$; IR (KBr) v $\mathrm{cm}^{-1} 3170(\mathrm{NH}), 2950$ (CH-aliph), $2200(\mathrm{C} \equiv \mathrm{N})$, $1660(\mathrm{C}=\mathrm{O}) ;{ }^{1} \mathrm{H}$ NMR $\left(\mathrm{DMSO}_{-} \mathrm{d}_{6}\right) \delta=2.52(\mathrm{~s}$, $\left.3 \mathrm{H}, \mathrm{CH}_{3}\right), 7.33-8.02(\mathrm{~m}, 9 \mathrm{H}, \mathrm{Ar}-\mathrm{H}$ and $\mathrm{NH}), 9.97$ (s, 1H, NH). Found; C, 57.09; H, 3.13; N, 19.98; cald. For $\mathrm{C}_{20} \mathrm{H}_{13} \mathrm{ClN}_{6} \mathrm{OS}$ (420.88): C, 57.08; H, $3.11 ; \mathrm{N}, 19.97$.

N-1,3-Benzothiazol-2-yl-5-cyano-6-imino1 - (4-methoxy-phenyl) - 4-methyl-1,6dihydropyridazine-3-carboxamide (8b)
It was obtained as brown crystals from ethanol; yield $69 \%$; m.p. $238^{\circ} \mathrm{C}$; IR $(\mathrm{KBr}) \vee \mathrm{cm}^{-1} 3357$, $3300(2 \mathrm{NH}), 3082$ (CH-arom), $2196(\mathrm{C} \equiv \mathrm{N}), 1661$ $(\mathrm{C}=\mathrm{O})$. Found; $\mathrm{C}, 60.58 ; \mathrm{H}, 3.88 ; \mathrm{N}, 20.17$; cald. For $\mathrm{C}_{21} \mathrm{H}_{16} \mathrm{~N}_{6} \mathrm{O}_{2} \mathrm{~S}$ (416.46): C, 60.57; H, 3.87; N, 20.18 .

Preparation of Compounds (9a,b): General Procedure

$\operatorname{Method}(A)$

A mixture of $(2 \mathrm{a}, \mathrm{b})(0.01 \mathrm{~mole})$, malononitrile (0.02 mole) and ammonium acetate $(2.0 \mathrm{gm})$ was fused for 30 minutes at $140^{\circ} \mathrm{C}$. The reaction mixture was left to stand at room temperature and triturated with ethanol. The solid product so formed was collected by filtration and recrystallized from the proper solvent to give $(9 a, b)$.

\section{Method (B)}

A mixture of (8a,b) (0.01 mole), malononitrile $(0.01 \mathrm{~mole})$ and ammonium acetate $(2.0 \mathrm{gm})$ was fused for 30 minutes at $140^{\circ} \mathrm{C}$. The reaction mixture was left to stand and triturated with ethanol. The solid product so formed was collected by filtration and recrystallized from the proper solvent to give $(9 a, b)$.

6-Amino-8-(1,3-Benzothiazol-2-yl-amino)-2-(4chloro-phenyl)-3-imino-2,3-dihydrocinnoline4,7-dicarbonitrile (9a)

It was obtained as brown crystals from dioxane/ethanol; yield $75 \%$; m.p. $>300^{\circ} \mathrm{C}$; IR $(\mathrm{KBr}) v \mathrm{~cm}^{-1} 3400,3341\left(\mathrm{NH}_{2}\right), 3194(\mathrm{NH}), 2202$ $(\mathrm{C} \equiv \mathrm{N}) ;{ }^{1} \mathrm{H}$ NMR $\left(\mathrm{DMSO}-\mathrm{d}_{6}\right) \delta=5.72(\mathrm{~s}, 2 \mathrm{H}$, $\mathrm{NH}_{2}$ ), 7.54-8.17 (m, 11H, Ar-H and 2NH). Found; C, 58.92; H, 2.78; N, 23.91; cald. For $\mathrm{C}_{23} \mathrm{H}_{13} \mathrm{ClN}_{8} \mathrm{~S}$ (468.93): C, 58.91; H, 2.79; N, 23.90.

6-A mino-8-(1,3-Benzothiazol-2-ylamino)-3-imino-2-(4-methoxyphenyl)-2,3dihydrocinnoline-4,7-dicarbonitrile (9b)

It was obtained as brown crystals from dioxane/ethanol; yield $70 \%$; m.p. $>300^{\circ} \mathrm{C}$; IR $(\mathrm{KBr}) v \mathrm{~cm}^{-1} 3400,3350\left(\mathrm{NH}_{2}\right), 3170(\mathrm{NH}), 2200$ $(\mathrm{C} \equiv \mathrm{N}) ; \mathrm{MS}: \mathrm{m} / \mathrm{z} 462\left(\mathrm{M}^{-2}\right)$. Found; $\mathrm{C}, 62.08 ; \mathrm{H}$, 3.48; N, 24.13; cald. For $\mathrm{C}_{24} \mathrm{H}_{16} \mathrm{~N}_{8} \mathrm{OS}$ (464.51): C, 62.06; H, 3.47; N, 24.12.

Preparation of Compounds (12a,b), (13): General Procedure

Equimolar amounts of (2b) (0.01 mole), arylidine malononitrile or maleic anhydride (0.01 mole), acrylonitrile ( 0.01 mole) and ammonium acetate $(0.5 \mathrm{gm})$ were heated at $150^{\circ} \mathrm{C}$ for $2 \mathrm{hrs}$. 
The reaction mixture was left to cool, triturated with ethanol and poured into water. The solid product so formed was collected by filtration and recrystallized from the proper solvent to give (12a,b), (13).

3-Acetyl-N-1,3-benzothiazol-2-yl-5-[1-cyano-2(4-methoxy-phenyl)vinyl]-1-(4-methoxyphenyl)2,3-dihydro-1H-[1,2,4]-triazole-3-carboxamide (12a):

It was obtained as brown crystals from ethanol; yield $70 \%$; m.p. $230-32^{\circ} \mathrm{C}$; IR ( $\left.\mathrm{KBr}\right) \vee \mathrm{cm}^{-1} 3328$, 3114 (2NH), 2996, 2931, 2835 (CH-aliph), 2201 $(\mathrm{C} \equiv \mathrm{N}), 1662(\mathrm{C}=\mathrm{O}) ;{ }^{1} \mathrm{H} \mathrm{NMR}\left(\mathrm{CDCl}_{3}\right) \delta=2.33$ $\left(\mathrm{s}, 3 \mathrm{H}, \mathrm{COCH}_{3}\right), 3.79\left(\mathrm{~s}, 3 \mathrm{H}, \mathrm{OCH}_{3}\right), 3.87(\mathrm{~s}, 3 \mathrm{H}$, $\left.\mathrm{OCH}_{3}\right), 5.93(\mathrm{~s}, 1 \mathrm{H}, \mathrm{CH}), 6.82-7.98(\mathrm{~m}, 13 \mathrm{H}, \mathrm{Ar}-\mathrm{H}$ and $\mathrm{NH}), 8.77$ (s, 1H, NH). Found; C, 63.04; H, 4.39 ; N, 15.23; cald. For $\mathrm{C}_{29} \mathrm{H}_{24} \mathrm{~N}_{6} \mathrm{O}_{4} \mathrm{~S}$ (552.62): C, 63.03; H, 4.38; N, 15.21

3-Acetyl-N-1,3-benzothiazol-2-yl-5-[2-(4chlorophenyl)-1-cyano-vinyl]-1-(4-methoxyphenyl)-2,3dihydro-1H-[1,2,4]-triazole-3-carboxamide (12b)

It was obtained as brown crystals from benzene; yield $76 \%$; m.p. $157-58^{\circ} \mathrm{C}$; IR (KBr) v $\mathrm{cm}^{-1}$ 3341, 3187 (2NH), 2950 (CH-aliph), 2204 $(\mathrm{C} \equiv \mathrm{N}), 1650(\mathrm{C}=\mathrm{O}) ;{ }^{1} \mathrm{H}$ NMR $\left(\mathrm{CDCl}_{3}\right) \delta=2.52$ $\left(\mathrm{s}, 3 \mathrm{H}, \mathrm{COCH}_{3}\right), 3.79\left(\mathrm{~s}, 3 \mathrm{H}, \mathrm{OCH}_{3}\right), 5.83(\mathrm{~s}, 1 \mathrm{H}$, $\mathrm{CH})$, 6.90-7.91 (m, 12H, Ar-H), 9.90 (s, 1H, NH), 12.77 (s, 1H, NH). Found; C, 60.39; H, 3.82; $\mathrm{N}, 15.08$; cald. For $\mathrm{C}_{28} \mathrm{H}_{21} \mathrm{ClN}_{6} \mathrm{O}_{3} \mathrm{~S}$ (557.03): C, $60.38 ; \mathrm{H}, 3.80 ; \mathrm{N}, 15.09$.

3-Acetyl-N-1,3-benzothiazol-2-yl-1-(4methoxyphenyl)-4,6-dioxo-hexahydro-furo [3,4-c] pyrazole-3-carboxamide (13)

It was obtained as brown crystals from DMF/ EtOH; yield $72 \%$; m.p. $>300^{\circ} \mathrm{C}$; IR $(\mathrm{KBr}) \vee \mathrm{cm}^{-1}$ 3375, 3201 (2NH), 2850 (CH-aliph), 1710, 1649 $(\mathrm{C}=\mathrm{O})$. Found; $\mathrm{C}, 56.66$; H, 3.90; N, 12.03; cald. For $\mathrm{C}_{22} \mathrm{H}_{18} \mathrm{~N}_{4} \mathrm{O}_{6} \mathrm{~S}$ (466.48): C, 56.65; H, 3.89; N, 12.01 .

5 - (1,3-Benzothiazol-2-yl-imino) - 3 methylisoxazol-4-(5H)-one-(4-methoxyphenyl) hydrazone (14)

A mixture of (2b) (0.01 mole), hydroxylaminehydrochloride $(0.01$ mole $)$ and sodium acetate $(0.5 \mathrm{~g})$ in ethanol $(30 \mathrm{ml})$ was treated with $1 \mathrm{ml}$ of water and heated under reflux for $8 \mathrm{hrs}$. The solid product formed after cooling was collected by filtration and recrystallized from ethanol to give $(14 ; 77 \%)$ as brown crystals, m.p. $230^{\circ} \mathrm{C}$; IR $(\mathrm{KBr}) \vee \mathrm{cm}^{-1} 3470(\mathrm{NH}) ; 2990(\mathrm{CH}-$ Egypt. J. Chem. 61, No. 6 (2018) aliph); ${ }^{1} \mathrm{H}$ NMR (DMSO-d $) \delta=2.48(\mathrm{~s}, 3 \mathrm{H}$, $\left.\mathrm{CH}_{3}\right), 3.80\left(\mathrm{~s}, 3 \mathrm{H}, \mathrm{OCH}_{3}\right), 6.99-7.99(\mathrm{~m}, 8 \mathrm{H}$, Ar-H), 12.71 (s, 1H, NH). Found; C, 59.17; H, 4.16; N, 19.18; cald. For $\mathrm{C}_{18} \mathrm{H}_{15} \mathrm{~N}_{5} \mathrm{O}_{2} \mathrm{~S}$ (365.41): C, 59.16; H, 4.14; N, 19.17 .

\section{References}

1. Benson S. C., Gross J. L. and Synder J. K., J. Org. Chem., 55, 3257 (1990).

2. Thomas A., Chakraborty M., Iia H. and Junjappa H., Tetrahedron, 46, 577 (1990).

3. Wolff J. and Taddei M., Tetrahedron, 42, 4267 (1986).

4. Taylor E. C., J. Heterocycl. Chem., 27, 1 (1990).

5. Tominaga Y., Kohra S., Honkawa H. and Hosomi A., Heterocycles, 29, 1409 (1989).

6. Hahn E. , In Lectures in "Heterocyclic Chemistry, IX”; Castle, R. N. Ed., p. 13 (1990).

7. Sanger F., Nicklen S. and Coulson A. R., Proc. Nitl. Acad. Sci. USA, 74, 5463 (1977).

8. Yamasaki T., Kawaminami E., Uchimura F., Okamoto Y., Okawara T. and Furukawa M., $J$. Heterocycl. Chem., 29, 825 (1992).

9. Kaji K., Nagashima H., Nagao S., Tabashi K. and Oda H., Chem. Pharm. Bull., 32, 4437 (1984).

10. Yamasaki T., Yoshihara Y., Okamoto Y., Okawara T. and Furukawa M., J. Heterocycl. Chem., 29, 1313 (1992).

11. Chen S. and Panzica R. P., J. Org. Chem., 46, 2467 (1981).

12. Elnagdi M. H..; Erian A. W., Liebigs Ann. Chem., 1225 (1990).

13. Elnagdi M. H., Abdelrazek F. M., Ibrahim N. S and Erian A. W., Tetrahedron, 45, 3597 (1989).

14. Elnagdi M. H., Negm A. M. and Erian A. W., Liebigs Ann. Chem., 1255 (1989).

15. Alzayed K. M. and Hafez E. A., J. Chem. Res., (S) 360, (M)1621 (1999).

16. Hussein A. M., Elnagdi M. H., Harb A. A. and Metwelly S. A., J. Prakt. Chem., 334, 723 (1992).

17. Hussein A. M., Atalla A. A., Abdel Hafez I. S. and Elnagdi M. H., Heteroatom Chemistry, 7, 6 (1996). 
18. Hussein A. M., Shrief S. M. and Atalla A. A., Monatshefte Fur Chemie, 127, 1153 (1996).

19. Christopher P. P., Adam L. G., Jeff W. K. and Adam J. M., Crystal Growth Design, 3 (6), 10211025 (2003).

20. Aziz S. I., Anwar H.F., El-Apasery M. A., Elnagdi M. H., J. Heterocycl. Chem., 44 (4), 877-881
(2007).

21. El-Adasy A., Kamel M. M., Saleh M. O., Hussein A. M., El-Apasery M. A., Int. J. ChemTech Res, 9 (6), 31- 38 (2016).

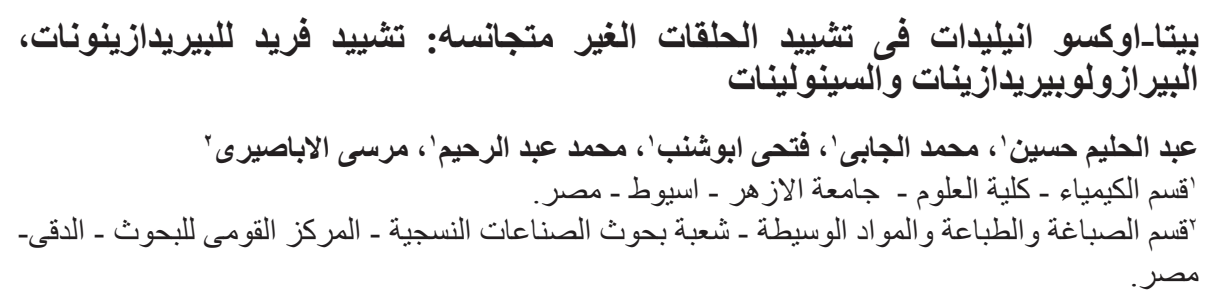

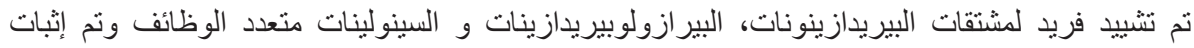

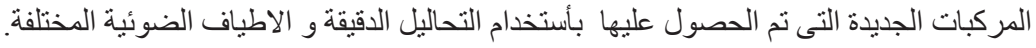

